\title{
ÍNDICE GEOMÉTRICO E PERDA DE CARGA LOCALIZADA EM CONEXÕES DE EMISSORES “ONLINE”
}

\author{
GABRIEL G. DE G. CARDOSO ${ }^{1}$, ANTÔNIO E. KLAR ${ }^{2}$
}

\begin{abstract}
RESUMO: O objetivo deste artigo é apresentar um estudo envolvendo uma relação entre a perda de carga localizada em conexões de emissores em tubulações, com diferentes diâmetros, utilizados em irrigação localizada, com a geometria dos conectores de emissores "online", que permite a fácil quantificação dessas perdas de carga, uma vez conhecida a dimensão dos conectores. O experimento foi conduzido para Números de Reynolds no intervalo de fluxo turbulento, obtidos pela variação da vazão nos tubos a uma temperatura constante da água. Para os tubos considerados, os resultados da investigação indicaram que o fator de atrito da equação de Darcy-Weisbach pode ser estimado pela Equação de Blasius com os coeficientes $b=0,300$ e $m=0,25$ para a determinação da perda de carga nos tubos avaliados. As perdas de carga provocadas pelas conexões dos emissores nos tubos considerados aumentaram em até $62 \%$ em relação às observadas nos tubos sem emissor. Uma relação entre o coeficiente de carga cinética $(K)$ e o índice de obstrução devido à conexão do emissor "online" (IO) no tubo é apresentada por uma relação algébrica do tipo potência, com coeficiente de ajuste de, aproximadamente, $96 \%$.
\end{abstract}

PALAVRAS-CHAVE: perda de carga localizada; equação de Blasius, Número de Reynolds.

\section{GEOMETRIC INDEX AND LOCAL HEAD LOSS IN CONNECTION OF "ONLINE" EMITTERS}

\begin{abstract}
The objective of this research is to present a study on a relationship between the local head loss in connection of emitters in pipes with different diameters used in drip irrigation, with the "online" geometry of the emitter connectors, that allows an easy quantification of such head loss regarding of the size of the connectors. The experiment was carried out according to the Reynolds Numbers at a turbulent flow interval, obtained by the variation of the pipe outflow at a constant temperature of water. The results indicated that the friction factor of the Darcy-Weisbach equation can be estimated by the Blasius equation with the coefficients $b=0.300$ and $m=0.25$, for the above mentioned pipes. The head losses produced by the connections of the emitters, in relation to the pipe without emitter, was of $62 \%$. A relationship between the kinetic load coefficient $(K)$ and the index of blockage $(I O)$ provoked by the "online" connector is presented by an algebraic equation which shows a coefficient of adjustment of approximately $96 \%$.
\end{abstract}

KEYWORDS: local head loss, Blasius equation, Reynolds number.

\section{INTRODUÇÃO}

$\mathrm{Na}$ irrigação localizada, o uso de gotejadores ou mesmo de microaspersores requer conexões,que são encaixes introduzidos na linha lateral, e lá permanecem ocupando uma área interna desta linha lateral, provocando assim uma perda de carga localizada (CARDOSO, 2007). A perda de carga localizada, causada pelo conector do emissor ou pelo próprio emissor no interior da tubulação, depende da área que o conector ou o emissor ocupa internamente na tubulação e de sua forma geométrica. Em função disso, os emissores são, comumente, classificados quanto ao tipo de conexão na tubulação: sobre a linha (online), na linha (in-line) e emissores integrados dentro da linha.

\footnotetext{
${ }^{1}$ Tecg $^{0}$ Irrigação e Drenagem, Prof. Doutor, Departamento de Agronomia, IFGoiano/Ceres - GO, Fone: (64) 3307-7100, gabriel.cardoso@ifgoiano.edu.br.

2 Eng ${ }^{\circ}$ Agrícola, Prof. Doutor, Departamento de Engenharia Agrícola, UNESP/FCA/Botucatu - SP, klar@fca.unesp.br. 
CARDOSO et al. (2007) propuseram equações, através de modelagem matemática, para calcular a perda de carga localizada provocada por emissores inseridos e integrados no tubo. Os modelos basearam-se no teorema de Bélanger, utilizando relações geométricas do tubo e dos emissores.

A perda de carga unitária ou gradiente de perda de carga, em uma tubulação hidraulicamente lisa e sem emissores conectados a ela, pode ser determinada com mais precisão, para qualquer regime hidráulico, pela equação geral de Darcy-Weisbach [eq. (1)] explicitada em função da velocidade média da água $V$; [eq. (1a)] ou da vazão $Q$; [eq. (1b)], também conhecida como equação universal de perda de carga.

$$
J=f \frac{1}{D} \frac{V^{2}}{2 g}
$$

ou

$$
J=\frac{8}{\pi^{2} g} f \frac{Q^{2}}{D^{5}}
$$

Para as tubulações comumente utilizadas como linha lateral em irrigação localizada, em função das vazões utilizadas e dos diâmetros internos, o Número de Reynolds $(R)$ se encontra, normalmente, na faixa nessas tubulações se encontram normalmente na faixa entre 2000 e $10^{5}$ $\left(2000<R<10^{5}\right)$. Nessa faixa, $f$ pode ser calculado com precisão por meio da equação de Blasius [eq. (2)]:

$$
f=0,316 R^{-0,25}
$$

em que, nas Eq. 1 e 2:

$J$ - gradiente de perda de carga na tubulação sem emissores $\left(\mathrm{m} \mathrm{m}^{-1}\right)$;

$f$ - fator de atrito;

$D$ - diâmetro interno da tubulação (m);

$V$ - velocidade média do fluxo na tubulação $\left(\mathrm{m} \mathrm{s}^{-1}\right)$;

$Q$ - vazão $\left(\mathrm{m}^{3} \mathrm{~s}^{-1}\right)$;

$g$ - aceleração da gravidade $=9,81 \mathrm{~m} \mathrm{~s}^{-2}$,

$R$ - número de Reynolds (adimensional).

Quando $R$ é muito elevado, a precisão é reduzida, pois o fator de atrito aproxima-se de zero. Quando $R<4 \times 10^{3}$, em regime laminar ou zonas críticas, a equação de Blasius superestima o fator de atrito. Para o escoamento na zona de regime hidráulico de transição, $2 \times 10^{3}<R<3 \times 10^{3}$, o fator de atrito pode ser aproximado por um valor constante, igual a 0,04 (YITAYEW, 2009).

O modelo da [eq. (2)] de Blasius pode ser apresentado como um modelo geral, conforme apresentado na [eq.(3)]:

$$
f=b R^{-m}
$$

em que:

$b$ e $m$ - coeficientes do modelo;

$R-$ já definido para a Eq. 2.

Nas eqs.(2) e (3), o adimensional $R$ pode ser expresso para tubulações, em função da velocidade da água [eq. (4a)], ou da vazão [eq. (4b)] e do diâmetro interno da tubulação, como: 


$$
R=\frac{V D}{v}
$$

ou

$$
R=\frac{4}{\pi} \frac{Q}{D v}
$$

em que:

$V, Q$ e $D$ - já definidos para a [eq. (1)];

$v$ - viscosidade cinemática da água em função da temperatura $\left(\mathrm{m}^{2} \mathrm{~s}^{-1}\right)$.

Com a inserção de um emissor na tubulação da linha lateral, a perda de carga localizada $\left(h f_{L}\right)$ devido à presença no interior da tubulação do conector do emissor (Figura 1), deve-se à resistência à movimentação da corrente fluida oferecida pela protrusão do conector no interior do tubo, podendo ser expressa como uma fração $K$ da carga cinética, obtida pelo princípio da similaridade de Reynolds [eq. (5)]:

$$
h f_{L}=K \frac{V_{o}^{2}}{2 g}
$$

em que:

$h f_{L}$ - perda de carga localizada devido à conexão do emissor (m);

$V_{o}$ - velocidade média de aproximação da corrente fluida $\left(\mathrm{m} \mathrm{s}^{-1}\right)$;

$K$ - coeficiente de carga cinética ou de resistência de perfil (adimensional);

$g$ - já definido para a [eq. (1)].

Analisando-se o esquema da Figura 1, verifica-se que a perda de carga localizada devida ao conector do emissor no interior da tubulação aumenta com o aumentando da velocidade de escoamento, uma vez que a turbulência do fluido, na passagem entre o elemento obstrutor e a parede do tubo, tende a aumentar.

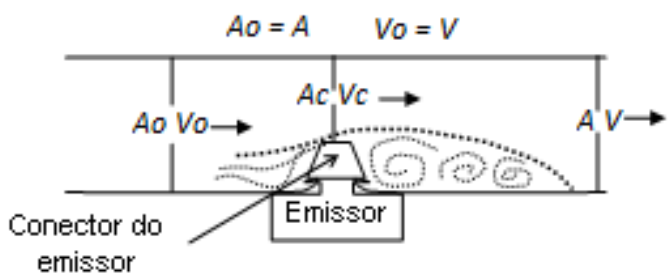

FIGURA 1. Contração e expansão do fluxo na conexão do emissor "online”. Contraction and expansion flow in the local connection of the "online" send.

Na Figura 1, tem-se: $A_{o}$ - área da seção interna normal, transversal ao eixo longitudinal da tubulação, antes do ponto de conexão do emissor na linha; $A_{c}$ - área de constrição: área da seção interna transversal ao eixo longitudinal, reduzida pela presença da altura do conector do emissor no interior da tubulação, no ponto de conexão; $A$ - área da seção interna normal da tubulação, transversal ao eixo longitudinal da tubulação, imediatamente após o ponto de conexão; $V_{o}-$ velocidade média do fluxo na seção $A_{o} ; V_{c}$ - velocidade média do fluxo na seção $A_{c} ; V$ - velocidade média do fluxo na seção $A$.

Segundo RETTORE NETO et al. (2009), as perdas localizadas nos conectores dos emissores “on-line” podem ser estudadas aplicando-se a equação de Bélanger. Na Figura 1, esquematiza-se um típico modelo de contração após a seção de constrição $A_{c}\left(A_{c}=\eta A_{o}\right)$, sendo $\eta$ a razão de 
obstrução $\left(A_{c} / A_{o}\right)$. Aplicando-se os teoremas da conservação da energia e da conservação da massa entre as seções $A_{c}$ e $A_{o}$ (Figura 1), chega-se à equação de Bélanger [eq. (6)]:

$$
h f_{L}=\frac{\left(V_{c}-V\right)^{2}}{2 g}=\left(\frac{A_{0}}{A_{c}}-1\right)^{2} \frac{V^{2}}{2 g}
$$

Comparando-se as eqs. (5) e (6), verifica-se que são correspondentes, pois as velocidades $V_{o}$ e $V$ são iguais (Figura 1). Na segunda igualdade da [eq. (6)], o termo entre parênteses é o fator geométrico, denominado índice de obstrução $(I O)$, e é dado por:

$$
I O=\left(\frac{A_{0}}{A_{c}}-1\right)^{2}=\left(\frac{1-\eta}{\eta}\right)^{2}
$$

Uma função matemática pode ser ajustada de forma que o coeficiente de carga cinética $K$, para cada conjunto tubo-conexão, pode ser estimado a partir da razão de obstrução. CARDOSO \& FRIZZONE (2007) propuseram uma relação matemática do tipo $K=\lambda(I O)^{\psi}$ [eq. (8)], e também que os valores de $h f_{L}$ podem ser obtidos experimentalmente, de acordo com a [eq. (9)]:

$$
h f_{L}=\frac{J^{\prime}-J}{N} L
$$

em que:

$h f_{L-}$ perda de carga localizada na conexão dos emissores (m);

$J$ e $J^{\prime}$ - perda de carga unitária na linha sem emissores $(J)$ e com emissores vedados $\left(J^{\prime}\right)$, (m $\left.\mathrm{m}^{-1}\right)$

$N$ - número de emissores na linha lateral;

$L$ - comprimento da linha lateral (m).

Neste trabalho, foi desenvolvida uma relação empírica $K=\lambda(I O)$, utilizando-se de quatro tipos de conexão de emissores “online” utilizados em sistemas de irrigação localizada.

\section{MATERIAL E MÉTODOS}

Este trabalho foi conduzido no laboratório de irrigação do Departamento de Engenharia Rural - ESALQ/USP, utilizando tubos de polietileno de baixa densidade, com diâmetros internos de 10,0 $\mathrm{mm} ; 12,9 \mathrm{~mm} ; 16,1 \mathrm{~mm} ; 17,4 \mathrm{~mm}$ e $19,7 \mathrm{~mm}$, com $15 \mathrm{~m}$ de comprimento entre os pontos de medição de pressão $(L)$ e com a tubulação em nível. Com vistas à caracterização dessas tubulações, os diâmetros internos dos tubos bem como o comprimento dos conectores dos emissores, todos de conexão "online”, foram medidos pelo método volumétrico e conferidos com as medidas realizadas em projetor óptico, modelo HB400-2, fabricado pela Starret Precision Optical. A espessura média da parede de cada um dos tubos foi determinada a partir de 10 anéis tomados perpendicularmente ao eixo longitudinal da tubulação, e a mensuração, feita com o projetor óptico. Essas espessuras dos tubos foram determinadas com o objetivo de caracterizar a tubulação utilizada no experimento, uma vez que valores distintos do apresentado nesta pesquisa podem ser encontrados ao se fazer uso de tubos com espessura distinta, devido à resistência à dilatação mecânica que os tubos sofrem com o aumento da pressão interna. ensaios.

Na Tabela 1, são apresentadas as principais características dos tubos utilizados antes dos 
TABELA 1. Principais características dos tubos utilizados no experimento. Main characteristics of the pipes used in the experiment.

\begin{tabular}{cccccc}
\hline $\begin{array}{c}\text { Diâmetro } \\
\text { nominal } \\
(\mathrm{DN})\end{array}$ & $\begin{array}{c}\text { Pressão } \\
\text { nominal } \\
(\mathrm{PN})\end{array}$ & $\begin{array}{c}\text { Espessura da } \\
\text { parede } \\
(\mathrm{mm})\end{array}$ & $\begin{array}{c}\text { Diâmetro } \\
\text { interno } \\
(\mathrm{mm})\end{array}$ & $\begin{array}{c}\text { Geometria da } \\
\text { seção }^{1}\end{array}$ & Superfície interna \\
\hline 12 & 20 & 0,826 & 10,00 & $\begin{array}{c}\text { Levemente } \\
\text { Elíptica }\end{array}$ & Lisa e polida \\
15 & 40 & 1,180 & 12,99 & $\begin{array}{c}\text { Elíptica } \\
\text { Levemente }\end{array}$ & Lisa e polida \\
18 & 20 & 0,832 & 16,27 & Liśptica & Lisa e polida \\
20 & 40 & 1,320 & 17,36 & Circular & Lisa e polida \\
22 & 40 & 1,540 & 19,65 & Circular & Lisa e polida \\
\hline
\end{tabular}

1 - Geometria da seção dos tubos sob condições internas e externas de pressão atmosférica local e temperatura ambiente de $25^{\circ} \mathrm{C}$

Para se caracterizar a geometria de um conector "online”, BAGARELLO et al. (1997) sugerem que sejam obtidas as dimensões da conexão como se apresentam na Figura 2.

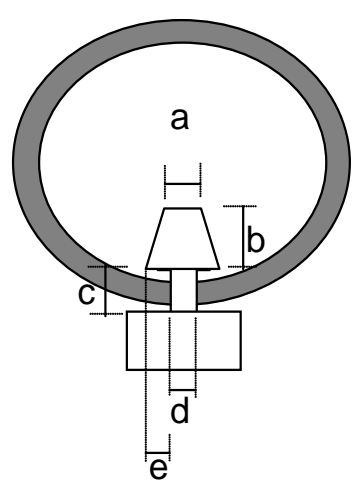

FIGURA 2. Caracterização das dimensões em conexão de emissores “online”. Characterization of dimensions in connection of online emitters.

Na Tabela 2, são apresentadas as dimensões médias de 10 conectores utilizados no experimento, realizada em projetor óptico, conforme o esquema da Figura 2.

TABELA 2. Característica dos emissores “online” utilizados. Characteristic of the online emitters used.

\begin{tabular}{ccccc}
\hline $\begin{array}{c}\text { Tipo - Modelo } \\
\text { Fabricante }\end{array}$ & $\begin{array}{c}\mathrm{C}_{1} \text { - Spray } \\
\text { Microjet / } \\
\text { Plasnova }\end{array}$ & $\begin{array}{c}\mathrm{C}_{2} \text { - Conector para } \\
\text { Microtubo / } \\
\text { Amanco }\end{array}$ & $\begin{array}{c}\mathrm{C}_{3} \text { - Click Tif-PC / } \\
\text { NaanDan }\end{array}$ & $\begin{array}{c}\mathrm{C}_{4} \text {-Katif / } \\
\text { Plastro }\end{array}$ \\
Dimensões & 3,932 & 3,368 & 3,006 & 2,142 \\
$\mathrm{a}(\mathrm{mm})$ & 3,186 & 5,070 & 6,125 & 5,187 \\
$\mathrm{c}(\mathrm{mm})$ & 3,254 & 4,949 & 3,499 & 2,636 \\
$\mathrm{~d}(\mathrm{~mm})$ & 4,360 & 4,417 & 4,989 & 6,505 \\
$\mathrm{e}(\mathrm{mm})$ & 1,627 & 1,525 & 1,129 & 0,962 \\
Volume $\left(\mathrm{mm}^{3}\right)$ & 61,893 & 86,980 & 89,250 & 122,778 \\
Área transversal $\left(\mathrm{mm}^{2}\right)$ & 19,700 & 23,590 & 26,500 & 31,630 \\
\hline
\end{tabular}

${ }^{1}$ Uso de produtos ou de marcas registradas tem a finalidade exclusiva de facilitar a compreensão.

O coeficiente de atrito ( $f$ ) foi expresso considerando-se três condições de uso da [eq. (3): a) com regressão considerando os coeficientes $b$ e $m$ da [eq. (3)], obtidos por regressão entre $R$ calculado com a [eq. (4)], e $f$ com a [eq. (1)] ( $f$ observado ajustado); b) considerando esses coeficientes iguais aos propostos por Blasius [eq. (2); $b=0,316$ e $m=0,25]$; c) com regressão 
considerando o coeficiente $m$ igual ao de Blasius $(0,25)$ e o $b$ obtido por regressão entre $R$ [eq. (4)] e $f$ [eq.(1)]. Para o cálculo de $f$ a partir dos dados experimentais, a [eq. (1b)] foi explicitada para $f$ como uma função de $J, D$ e $Q$. Para isso, os valores de $D$ foram os selecionados para este trabalho, os de $Q$ foram medidos e os de $J$ foram calculados com a [eq. (10)], como está apresentada a seguir:

$$
J=\frac{\Delta H}{L}
$$

A medição da perda de carga total $(\Delta H)$ ao longo do comprimento $L$ (15 m) para cada diâmetro testado foi realizada com um manômetro diferencial de coluna de mercúrio com menor leitura de $1 \mathrm{~mm}$ de altura. Essas medições foram realizadas a intervalos de 4 minutos para cada vazão testada, dentro da faixa de $R$ entre $6 \times 10^{3}$ e $7,2 \times 10^{4}$. Os valores de $J$ foram calculados com a Eq. 10, sendo que os valores de $\Delta H$ utilizados foram valores médios de três repetições de medidas de $Q$. Os valores de $J$, assim obtidos, foram utilizados também na [eq. (9)].

Para a determinação da perda de carga unitária nas tubulações com os emissores conectados e vedados ( $J$ '), foi utilizada a mesma metodologia já descrita para o cálculo de $J$, mas agora com as tubulações dotadas de emissores "online” conectados (emissores espaçados de 0,5 m; 29 emissores ao longo dos $15 \mathrm{~m}$ de tubulação). Para cada combinação diâmetro-emissor, $J$ ' foi calculado com a [eq. (11)] a seguir, considerando que $\Delta H$ 'é a perda de carga total na tubulação com os emissores conectados e vedados:

$$
J^{\prime}=\frac{\Delta H^{\prime}}{L}
$$

Os valores de $J$ ' obtidos pela [eq. (11)], assim como os de $J$ da [eq. (10)], foram substituídos na [eq. (9)] para a obtenção da perda de carga localizada adicional causada pela presença do conector do emissor no interior da tubulação $\left(h f_{L}\right)$.

Durante os ensaios, a pressão da água na entrada do tubo variou entre 150 e $300 \mathrm{kPa}$, e a temperatura média da água foi de $20{ }^{\circ} \mathrm{C}$, medida com um termômetro digital. Durante o ensaio, a água bombeada para a tubulação de teste não retornava para o reservatório de $20 \mathrm{~m}^{3}$ de onde provinha, minimizando variações na temperatura devido ao aquecimento no bombeamento. Como o ensaio foi realizado com as tubulações colocadas em nível, considerou-se a perda de carga total no tubo, tanto para $\Delta \mathrm{H}$ como para $\Delta \mathrm{H}^{\prime}$, como sendo a diferença de pressão entre as duas extremidades, distantes $15 \mathrm{~m}$ entre a tomada no início e o final da linha.

A vazão foi controlada com um registro de gaveta acoplado ao final da tubulação e medida com um medidor de vazão eletromagnético, modelo KC1000, associado a um conversor de sinais IFC010, ambos fabricados pela empresa Controles Automáticos Ltda. (CONAUT). Desta forma, a vazão de entrada no tubo era a mesma de saída, não havendo descarga de água através dos emissores, uma vez que estes foram desmontados e vedados internamente com veda-calha. De acordo com as informações do fabricante, esse aparelho apresenta capacidade de leitura de 2,78 $\mathrm{x}$ $10^{-7} \mathrm{~m}^{3} \mathrm{~s}^{-1}\left(1,0 \mathrm{~L} \mathrm{~h}^{-1}\right)$ e precisão de $\pm 0,14 \%$, para vazões compreendidas entre $5,56 \mathrm{x} 10^{-5}$ e $5,56 \mathrm{x}$ $10^{-1} \mathrm{~m}^{3} \mathrm{~s}^{-1}$ (200 a $2.000 \mathrm{~L} \mathrm{~h}^{-1}$ ), intervalo este que é maior que o intervalo de vazões relativas à faixa de Número de Reynolds testada $\left(6 \times 10^{3}\right.$ a $\left.7,2 \times 10^{4}\right)$.

Uma bomba hidráulica centrífuga com motor elétrico acoplado foi usada para impulsionar a água no circuito a partir de um reservatório subterrâneo. Na Figura 3, é mostrado o esquema de funcionamento do ensaio de perda de carga, com medidor de vazão eletromagnético acoplado à tubulação de teste, juntamente com o manômetro diferencial. Para cada variação de perda de carga aferida, um valor de vazão pôde ser aferido instantaneamente pelo medidor eletromagnético. 


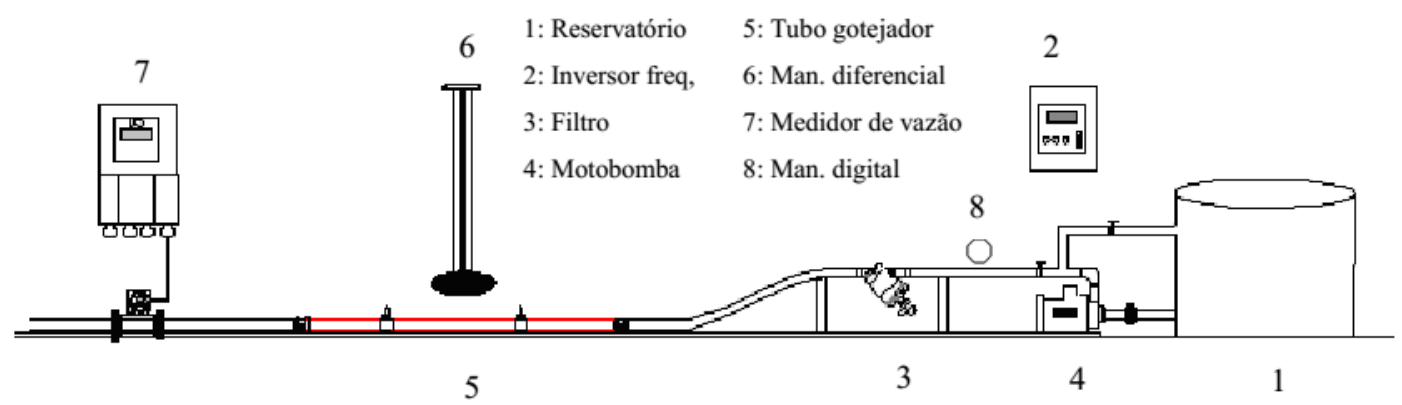

FIGURA 3. Esquema sem escala do circuito hidráulico experimental utilizado para a determinação da perda de carga. Scheme without scale of experimental hydraulic circuit used for determining the head loss.

As perdas de carga unitárias observadas nas tubulações com emissores instalados eqs. (10) e (11), respectivamente, em função das vazões, foram utilizadas também para, através de análise de regressão, determinar o coeficiente $\beta$ do modelo tipo potência da [eq. (12)]:

$$
J^{\prime}=\beta Q^{n}
$$

em que:

$J$ ' - já definido na [eq. (9)];

$Q$ - vazão de entrada da tubulação $\left(\mathrm{m}^{3} \mathrm{~s}^{-1}\right)$,

$\beta$ e $n$ - coeficientes do modelo.

Para que houvesse possibilidade de comparação com a equação de Darcy-Weisbach, com $f$ calculado por Blasius [eq. (2)], foram ajustadas equações fixando-se o coeficiente $n$ da [eq. (12)] em 1,75 , que é o mesmo expoente da vazão $(Q)$ na [eq. (1b)], quando se substitui na [eq. (1)] a [eq. (2)], e nesta a [eq. (4b)]. Assim, para cada diâmetro e considerando a viscosidade cinemática da água para a temperatura de $20{ }^{\circ} \mathrm{C}\left(1,01 \times 10^{-6} \mathrm{~m}^{2} \mathrm{~s}^{-1}\right)$, a [eq. (1b)] pode ser reescrita originando a [eq. (13)] a seguir:

$$
J=7,7910^{-4} \frac{Q^{1,75}}{D^{4,75}}
$$

O acréscimo percentual de perda de carga devido à inserção dos conectores dos emissores nas tubulações, em relação às sem emissor, foi calculado pela diferença dos valores entre as perdas de carga nos tubos com emissores $J$ ' [eq. (11)] e as perdas de carga nos tubos sem emissores $J$ [eq. (10)]. Com esta diferença, fez-se a relação percentual com a perda de carga no tubo sem emissor $(J)$ com a expressão: $100\left[\left(J^{\prime}-J\right) / J\right]$.

Fazendo uso das eqs. (9), (10) e (11), foi possível determinar as perdas de carga localizadas por emissor $\left(h f_{L}\right)$, para todos os modelos de emissores, para $15 \mathrm{~m}$ de comprimento da linha $(L)$ e 29 emissores $(N)$ conectados na linha ao longo desse comprimento. Os valores destas perdas foram correlacionados aos valores de $\mathrm{V}^{2} / 2 \mathrm{~g}$, gerando um coeficiente $K$ como parâmetro da [eq. (5)]. Para cada combinação tubo-conexão, tem-se um valor médio de $K$, valor este correlacionado aos valores do índice de obstrução (IO) obtidos com a [eq. (7), dando origem a uma equação do tipo da [eq. (8)]. Assim, a perda de carga localizada $h f_{L}$ pôde ser determinada, fazendo-se uso desse índice geométrico, como na [eq. (5)]. 


\section{RESULTADOS E DISCUSSÃO}

Na Figura 3, estão apresentadas as curvas do coeficiente de atrito - $f$ em função do Número de Reynolds $-R$, para os dados experimentais, e para o modelo de Blasius [eqs. (2) e (3) com $m=0,25]$. Considerando todos os diâmetros analisados, os valores dos coeficientes b e m da [eq. (3), o valor de $m$ foi de 0,273 para ajustar aos dados observados. O valor de $b$ foi de 0,377. Fixando-se o valor de $m$ em 0,25 , o valor de $b$ resultou igual a 0,300 , enquanto VON BERNUTH (1989) encontraram para este parâmetro um valor de 0,345 para tubos de PVC; e para tubos de polietileno, BAGARELLO et al. (1995) obtiveram $b$ igual a 0,302, contra 0,316 utilizado na equação de Blasius. Todos os pares $(R, f)$ das funções ajustadas localizam-se abaixo da curva de Blasius (Figura 4), concordando com as observações feitas por BAGARELLO et al. (1995). Este fato pode ser justificado pelo aumento de diâmetro dos tubos de polietileno quando submetidos à pressão (VILELA et al., 2003) e porque, segundo justificam BAGARELLO et al. (1995), os atuais tubos de polietileno apresentam paredes internas mais lisas e polidas que os tubos utilizados por Blasius.

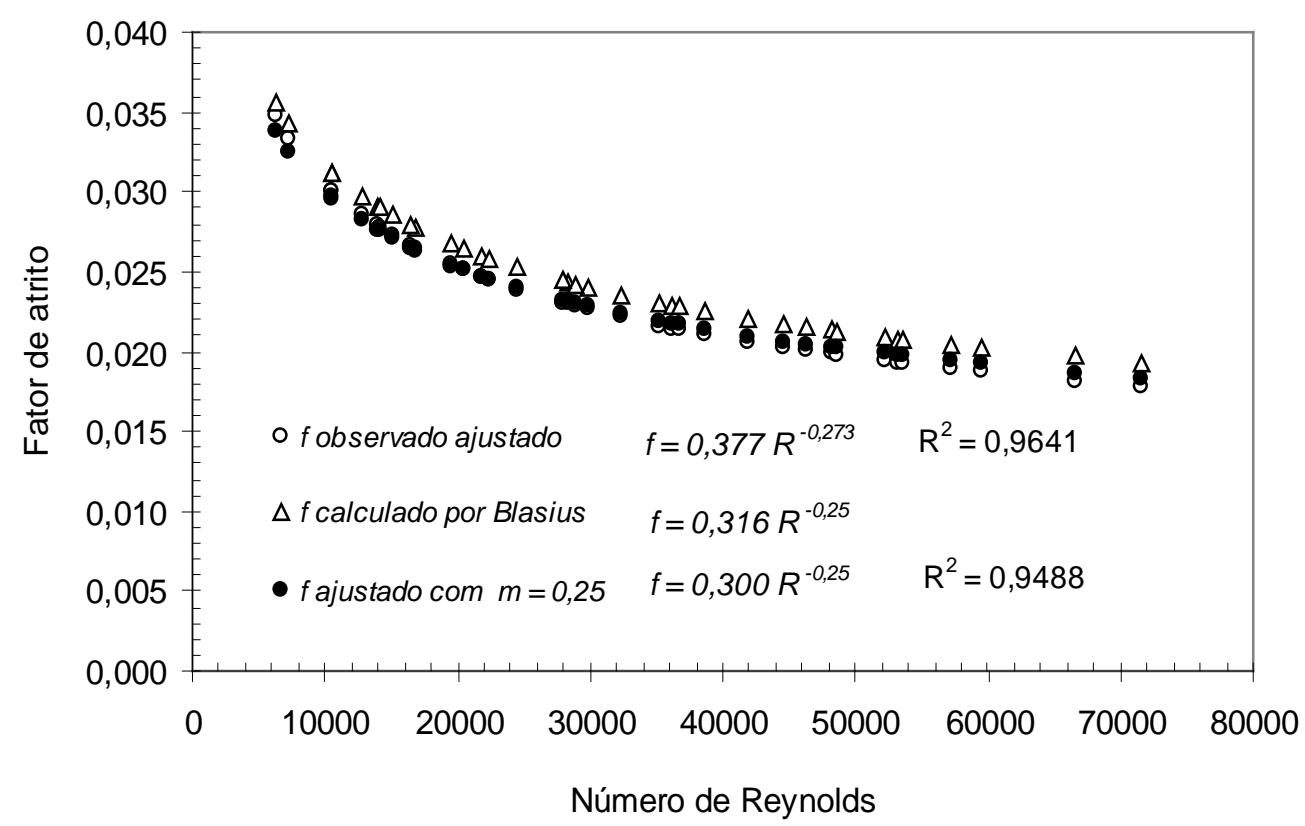

FIGURA 4. Curvas de fator de atrito $(f)$ em função do Número de Reynolds $(R)$, obtidas com o ajuste aos dados experimentais ( $f$ observado ajustado), com a equação de Blasius ( $f$ calculado por Blasius) e com ajuste aos dados experimentais com $m=0,25$ ( $f$ ajustado com $m=0,25$ ). Curves of factor of attrition (f) as a function of Reynolds number ( $R$ ), for the adjustment to experimental data (f observed adjusted), with Blasius equation (f calculated by Blasius)and the adjustment to the experimental data with $\mathrm{m}=0,25$ (f adjusted with $\mathrm{m}=\mathbf{0 , 2 5}$ ).

Na Figura 5, apresenta-se a concordância entre os valores de $f$ calculados pela equação de Blasius [eq. (2); $b=0,316$ e $m=0,25$ ] e os valores experimentais de $f$ obtidos com os valores de $J$ das [eq. (10)] inseridos na [eq. (1)]. Na Figura 6, a concordância é entre os valores de $f$ calculados com a equação de Blasius [eq. (2)] e os ajustados com $m=0,25$ e $b=0,300$. Verifica-se, na Figura 5 , que os valores obtidos estão distribuídos, na maioria dos casos, acima da reta 1:1, exceto para o diâmetro de 10 mm (Figura 5), indicando que a equação de Blasius superestima os valores de $f$ para os diâmetros superiores a 10,0 mm. Na Figura 6, observa-se que os valores de $f$ ajustados para $m=$ 0,25 estão mais próximos daqueles calculados pela equação de Blasius ( $b=0,316$ e $m=0,25$ ), porém sempre mais inferiores a estes, do que com os calculados pela equação de $f$ observado ajustado ( $b=0,377$ e $m=0,273$ ). A maior diferença percentual entre os valores de $f$ observados e os valores de $f$ de Blasius foi de 10,26\% e a menor foi 0,20\%, sendo, em média, 5,6\%, e 42\% das 
diferenças foram inferiores a 5\%. Já, a diferença média entre os valores de $f$ calculados por Blasius e $f$ calculados pela equação ajustada com $m=0,25$ foi $5,1 \%$. Para ambos os casos, o índice de concordância de Willmoth $d$ (WILLMOTH, 1981), o índice de correlação de Pearson $r$ (MONTGOMERY \& RUNGER, 2003) e o índice de confiança de Camargo c (CAMARGO \& SENTELHAS, 1997) indicaram concordância estreita entre os valores de $f$ observados e os ajustados com $m=0,25$ ao modelo de Blasius. Desses resultados, pode-se indicar como melhor alternativa para o cálculo do fator de atrito, nos tubos analisados, a equação: $f=0,300 R^{-0,25}$, concordando com os estudos experimentais e semiteóricos conduzidos por BAGARELLO et al. (1995) que os levaram a propor $b=0,302$. A importância em se adotar uma equação do fator de atrito, com expoente semelhante à equação de Blasius, está na possibilidade de usá-la num modelo de perda de carga e compará-lo com a equação de Darcy-Weisbach com fator de atrito de Blasius.

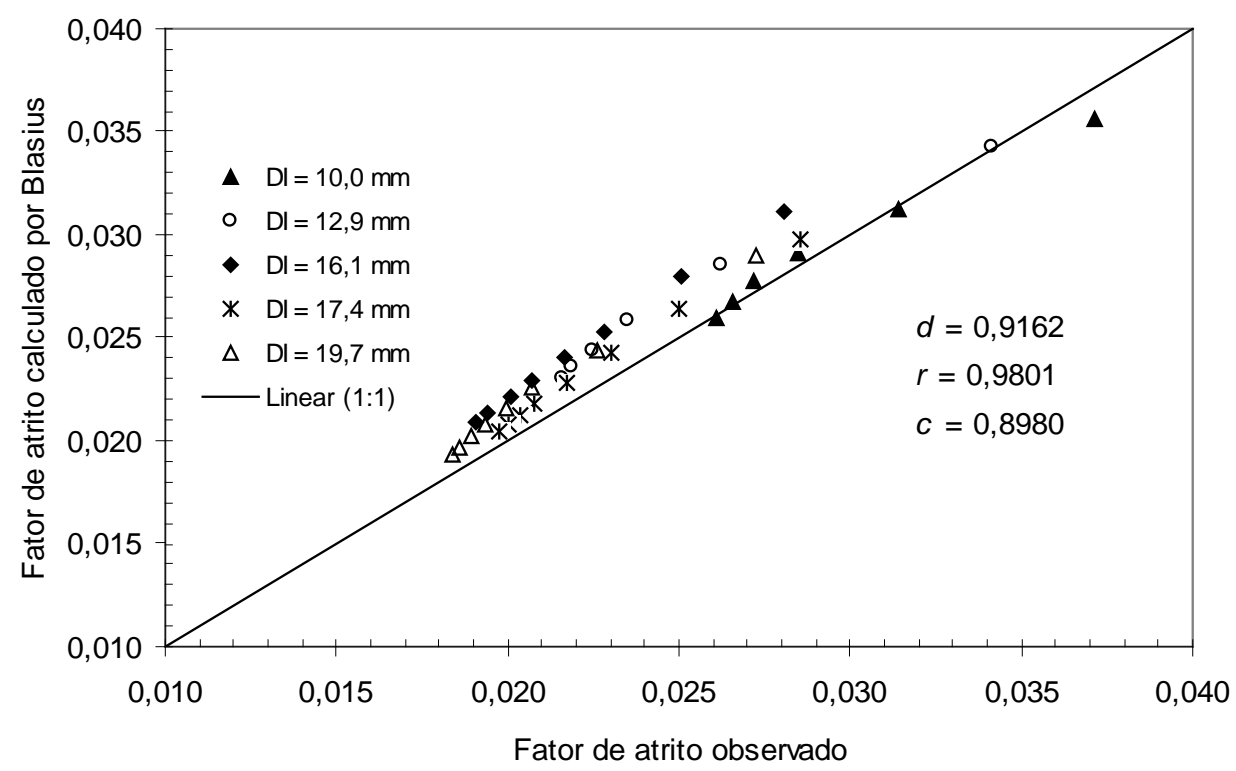

FIGURA 5. Concordância entre os valores de $f$ observados e os valores calculados pela equação de Blasius. Comparison between the observed values of $\mathbf{f}$ and the values calculated by the Blasius equation.

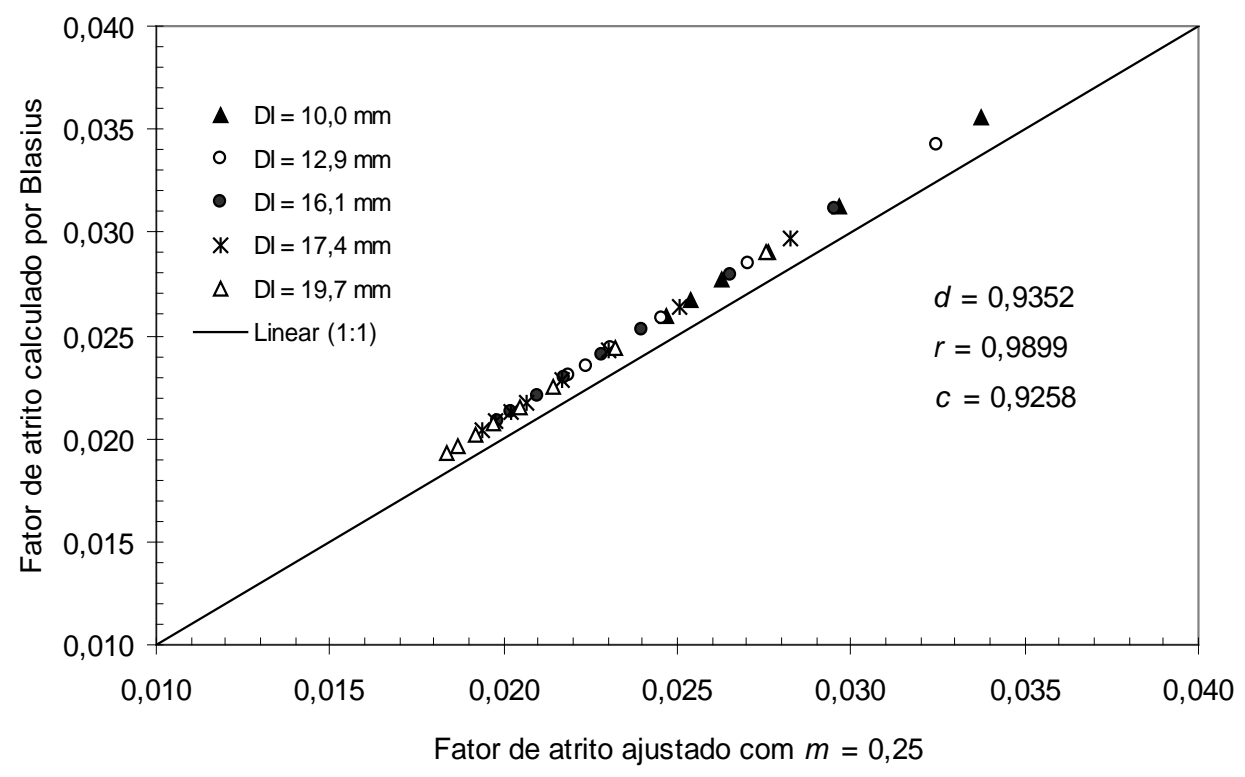

FIGURA 6. Concordância entre os valores de $f$ ajustados com $m=0,25$ e os valores calculados pela equação de Blasius. Agreement between the values of $\mathbf{f}$ with $\mathbf{m}=\mathbf{0 , 2 5}$ and values calculated by the Blasius equation. 
Com a determinação do fator de atrito $f$, utilizando-se de $m=0,25$ e $b=0,300$ e substituindo a [eq. (4)] na [eq. (3)] com esses coeficientes, e esta na [eq. (1)], a equação de Darcy-Weisbach [eq. (1)] pôde ser reescrita de forma a representar a perda de carga distribuída e unitária da tubulação com emissores conectados e vedados $\left(J^{\prime} ; \mathrm{m} \mathrm{m}^{-1}\right)$, em função da vazão $Q\left(\mathrm{~m}^{3} \mathrm{~s}^{-1}\right)$, do diâmetro do tubo $D(\mathrm{~m})$ e da viscosidade cinemática $v\left(\mathrm{~m}^{2} \mathrm{~s}^{-1}\right)$, conforme a [eq. (14)]:

$$
J^{\prime}=2,333610^{-2} v^{0,25} Q^{1,75} D^{-4,75}
$$

Na Tabela 3, são apresentados os valores do coeficiente $\beta$ da [eq. (11)], para a determinação das perdas de carga unitárias nos tubos com os conectores $\left(J^{\prime}\right)$, determinadas para os cinco diâmetros selecionados, combinados com os quatro modelos de conectores, em função da vazão na entrada da tubulação $(Q)$ e para o valor constante 1,75 do expoente $n$ de $Q$. Verifica-se que, em ordem decrescente de valores de $\beta$, e para todos os diâmetros testados, a conexão do emissor que provocou maior perda de carga, foi a do Katif-Plastro $\left(\mathrm{C}_{4}\right)$, seguida do Click Tif-PC-Naan Dan $\left(C_{3}\right)$, depois do microtubo Amanco $\left(C_{2}\right)$ e, finalmente, pela do Spray Microject-Plasnova $\left(C_{1}\right)$. Observando os volumes, bem como as áreas dos conectores representados na Tabela 2, confirma-se que, quanto maior a geometria de encaixe do emissor, maior é a perda de carga provocada pelo mesmo. Observa-se que as perdas de carga aumentaram com o aumento da área da seção transversal da protrusão do conector.

TABELA 3. Valores do coeficiente $\beta$ para o cálculo das perdas de carga unitárias nos tubos com conectores de emissores “online” $\left(J^{\prime}\right)$, considerando $n=1,75$ da equação de Blasius.

Coefficient $\beta$ values to calculate the unitary head loss on pipes with connectors of emitters "online" $\left(J^{\prime}\right)$, considering Blasius equation with $\mathbf{n}=1.75$.

\begin{tabular}{cccc}
\hline $\begin{array}{c}\text { Diâmetro interno } \\
\text { do tubo } \\
(\mathrm{mm})\end{array}$ & Tipo de conector & Parâmetro $\beta$ de & $J^{\prime}=\beta Q^{1,75}$ \\
\hline $\mathrm{D}_{1}=10,0$ & & & $\mathrm{R}^{2}$ \\
& $\mathrm{C}_{1}$ & 2925926,9 & 0,9997 \\
& $\mathrm{C}_{2}$ & 3219434,7 & 0,9998 \\
& $\mathrm{C}_{3}$ & 3479912,3 & 0,9996 \\
$\mathrm{C}_{4}=13,0$ & $\mathrm{C}_{1}$ & 3777489,2 & 0,9985 \\
\hline $\mathrm{D}_{2}$ & $\mathrm{C}_{2}$ & 778260,5 & 0,9994 \\
& $\mathrm{C}_{3}$ & 874964,6 & 0,9976 \\
& $\mathrm{C}_{4}$ & 903177,9 & 0,9977 \\
$\mathrm{D}_{3}=16,3$ & $\mathrm{C}_{1}$ & 1010585,0 & 0,9972 \\
\hline & $\mathrm{C}_{2}$ & 258896,1 & 0,9997 \\
& $\mathrm{C}_{3}$ & 280262,7 & 0,9994 \\
& $\mathrm{C}_{4}$ & 294403,8 & 0,9999 \\
$\mathrm{D}_{4}=17,4$ & $\mathrm{C}_{1}$ & 338598,0 & 0,9988 \\
\hline & $\mathrm{C}_{2}$ & 190106,4 & 0,9996 \\
& $\mathrm{C}_{3}$ & 205342,7 & 0,9991 \\
& $\mathrm{C}_{4}$ & 212415,5 & 0,9993 \\
& $\mathrm{C}_{1}$ & 235126,5 & 0,9993 \\
\hline $\mathrm{D}_{5}=19,7$ & $\mathrm{C}_{2}$ & 104464,1 & 0,9994 \\
& $\mathrm{C}_{3}$ & 112235,9 & 0,9990 \\
& $\mathrm{C}_{4}$ & 115890,1 & 0,9991 \\
\hline
\end{tabular}

C1 - Spray Microjet/Plasnova; C2 - Conector para microtubo/Amanco; C3 - Click Tif-PC/NaanDan; C4 -Katif/Plastro.

Na Figura 7, apresenta-se o acréscimo percentual de perda de carga nos tubos, provocado pela conexão dos emissores em relação à tubulação sem emissores, em função do diâmetro dos tubos, 
para os quatro tipos de conexões estudadas. O maior acréscimo, em torno de 61,5\%, ocorreu para a conexão do emissor Katif, no tubo com diâmetro de $10 \mathrm{~mm}$, e o menor acréscimo, 10,5\%, para o conector do emissor Spray Microjet, instalado no tubo com diâmetro de 19,7. Observa-se que os conectores de maiores áreas da seção transversal apresentam maiores acréscimos de perda de carga, isso porque o coeficiente de perda de carga localizada, $K$, tende a aumentar para obstruções maiores no fluxo da água.

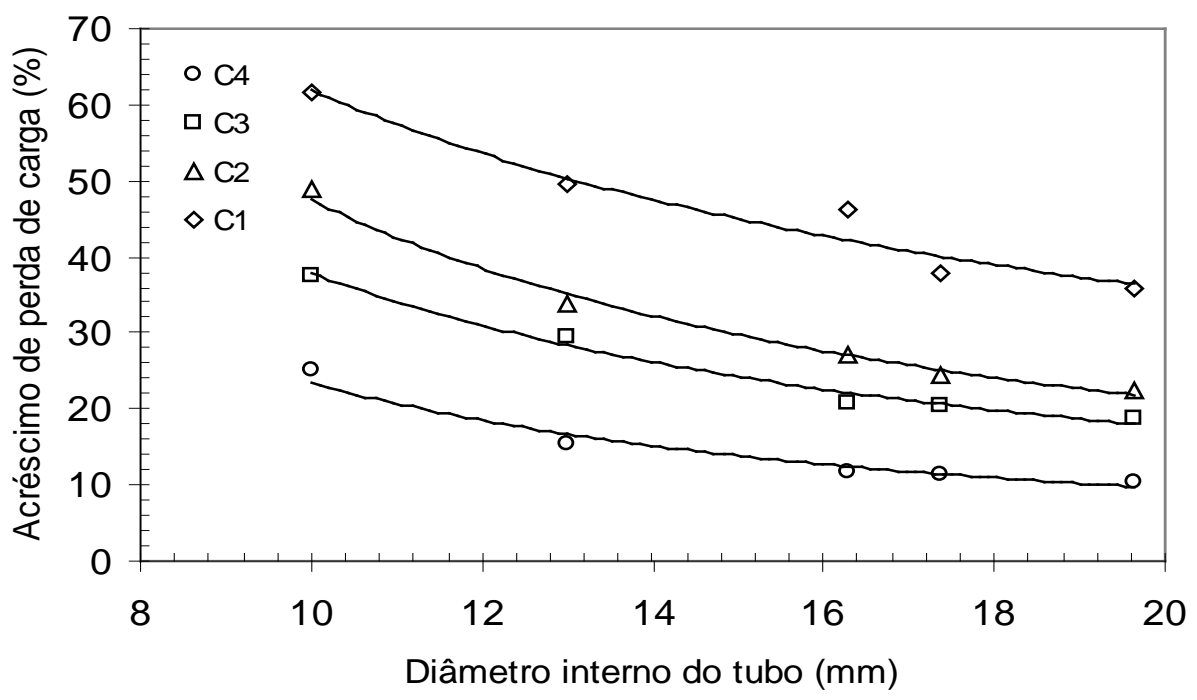

FIGURA 7. Acréscimo percentual de perda de carga devida à conexão dos emissores em função dos diâmetros dos tubos ensaiados. Percentage of increase head loss due to the connections of the emitters depend on the diameter of the assayed pipes.

Na Tabela 4, são apresentados os valores do coeficiente de carga cinética $(K)$ para cálculo da perda de carga localizada $\left(h f_{L}\right)$, em função de $V^{2} / 2 g$ para os quatro modelos de conectores instalados nos cinco diâmetros de tubos utilizados. Pode-se constatar que, para um mesmo diâmetro de tubo, as perdas de carga localizadas aumentam de acordo com a seguinte sequência de conexão: $\mathrm{C}_{4}>\mathrm{C}_{3}>\mathrm{C}_{2}>\mathrm{C}_{1}$, o que corresponde ao aumento da perda de carga localizada com o aumento da área da seção transversal do conector, com o consequente aumento do valor de K, de C1 para C4.

TABELA 4. Coeficientes de carga cinética $(K)$ para cálculo da perda de carga localizada nas conexões dos emissores. Kinetic head loss coefficients $(K)$ for calculation of the located head loss in the emitter connections.

\begin{tabular}{|c|c|c|c|}
\hline $\begin{array}{c}\text { Diâmetro interno } \\
\text { do tubo } \\
(\mathrm{mm})\end{array}$ & Tipo de conector & $\begin{array}{l}\text { Parâmetro } K \text { da eq. } \\
\qquad h f_{L}=K V^{2} / 2 g\end{array}$ & $\mathrm{R}^{2}$ \\
\hline \multirow{4}{*}{$\mathrm{D}_{1}=10,0$} & $\mathrm{C}_{1}$ & 0,3306 & 0,9913 \\
\hline & $\mathrm{C}_{2}$ & 0,4971 & 0,9918 \\
\hline & $\mathrm{C}_{3}$ & 0,6458 & 0,9920 \\
\hline & $\mathrm{C}_{4}$ & 0,8154 & 0,9912 \\
\hline \multirow{4}{*}{$\mathrm{D}_{2}=13,0$} & $\mathrm{C}_{1}$ & 0,1378 & 0,9942 \\
\hline & $\mathrm{C}_{2}$ & 0,2671 & 0,9942 \\
\hline & $\mathrm{C}_{3}$ & 0,3045 & 0,9940 \\
\hline & $\mathrm{C}_{4}$ & 0,4495 & 0,9933 \\
\hline \multirow{4}{*}{$\mathrm{D}_{3}=16,3$} & $\mathrm{C}_{1}$ & 0,0783 & 0,9926 \\
\hline & $\mathrm{C}_{2}$ & 0,1398 & 0,9927 \\
\hline & $\mathrm{C}_{3}$ & 0,1806 & 0,9923 \\
\hline & $\mathrm{C}_{4}$ & 0,3079 & 0,9923 \\
\hline \multirow{4}{*}{$\mathrm{D}_{4}=17,4$} & $\mathrm{C}_{1}$ & 0,0695 & 0,9933 \\
\hline & $\mathrm{C}_{2}$ & 0,1236 & 0,9935 \\
\hline & $\mathrm{C}_{3}$ & 0,1488 & 0,9933 \\
\hline & $\mathrm{C}_{4}$ & 0,2291 & 0,9937 \\
\hline \multirow{4}{*}{$\mathrm{D}_{5}=19,7$} & $\mathrm{C}_{1}$ & 0,0533 & 0,9926 \\
\hline & $\mathrm{C}_{2}$ & 0,0951 & 0,9925 \\
\hline & $\mathrm{C}_{3}$ & 0,1148 & 0,9928 \\
\hline & $\mathrm{C}_{4}$ & 0,1823 & 0,9925 \\
\hline
\end{tabular}


Com relação aos valores do índice de obstrução (IO), estes diminuem conforme aumenta a área da seção interna normal, transversal ao eixo longitudinal da tubulação, antes do ponto de conexão do emissor na linha $\left(A_{o}\right)$ e aumenta seu valor conforme o aumento da razão de obstrução $(\eta)$ (Tabela 5). O conector $\mathrm{C}_{4}$ apresentou maior índice de obstrução para todos os tubos analisados, seguido pelo conector $\mathrm{C}_{3}$, depois o $\mathrm{C}_{2}$ e, finalmente, pelo $\mathrm{C}_{1}$, devido à sua maior área transversal, conforme se verifica na Tabela 2.

TABELA 5. Variáveis geométricas e índice geométrico (IO) das tubulações em função dos diâmetros internos e dos tipos de conectores utilizados. Geometric variables and geometric index (IO) of pipes depending on the internal diameters and types of connectors used.

\begin{tabular}{ccccccc}
\hline \multirow{2}{*}{\begin{tabular}{c} 
Diâmetro interno $\begin{array}{c}c \\
\text { do tubo } \\
(\mathrm{mm})\end{array}$ \\
\cline { 3 - 6 }
\end{tabular}} & Tipo de conector & $\begin{array}{c}A_{o} \\
(\mathrm{~mm})\end{array}$ & Ac $(\mathrm{mm})$ & $\eta$ & IO & médio \\
\hline \multirow{2}{*}{$\mathrm{D}_{1}=10,0$} & $\mathrm{C}_{1}$ & 78,50 & 58,840 & 0,7492 & 0,1121 & \\
& $\mathrm{C}_{2}$ & 78,50 & 54,950 & 0,6996 & 0,1843 & 0,2526 \\
& $\mathrm{C}_{3}$ & 78,50 & 52,040 & 0,6626 & 0,2593 & \\
& $\mathrm{C}_{4}$ & 78,50 & 46,910 & 0,5973 & 0,4546 & \\
\hline \multirow{2}{*}{$\mathrm{D}_{2}=13,0$} & $\mathrm{C}_{1}$ & 132,67 & 112,828 & 0,8514 & 0,0305 & \\
& $\mathrm{C}_{2}$ & 132,67 & 108,938 & 0,8220 & 0,0469 & 0,0595 \\
& $\mathrm{C}_{3}$ & 132,67 & 106,028 & 0,8000 & 0,0625 & \\
& $\mathrm{C}_{4}$ & 132,67 & 100,898 & 0,7613 & 0,0983 & \\
\hline \multirow{2}{*}{$\mathrm{D}_{3}=16,3$} & $\mathrm{C}_{1}$ & 208,57 & 188,206 & 0,9052 & 0,0110 & \\
& $\mathrm{C}_{2}$ & 208,57 & 184,316 & 0,8865 & 0,0164 & 0,0202 \\
& $\mathrm{C}_{3}$ & 208,57 & 181,406 & 0,8725 & 0,0213 & \\
& $\mathrm{C}_{4}$ & 208,57 & 176,276 & 0,8479 & 0,0322 & \\
\hline \multirow{2}{*}{$\mathrm{D}_{4}=17,4$} & $\mathrm{C}_{1}$ & 237,67 & 216,996 & 0,9168 & 0,0082 & \\
& $\mathrm{C}_{2}$ & 237,67 & 213,106 & 0,9003 & 0,0123 & 0,0150 \\
& $\mathrm{C}_{3}$ & 237,67 & 210,196 & 0,8880 & 0,0159 & \\
& $\mathrm{C}_{4}$ & 237,67 & 205,066 & 0,8664 & 0,0238 & \\
\hline & $\mathrm{C}_{1}$ & 304,65 & 283,561 & 0,9350 & 0,0048 & \\
$\mathrm{D}_{5}=19,7$ & $\mathrm{C}_{2}$ & 304,65 & 279,671 & 0,9222 & 0,0071 & 0,0087 \\
& $\mathrm{C}_{3}$ & 304,65 & 276,761 & 0,9126 & 0,0092 & \\
& $\mathrm{C}_{4}$ & 304,65 & 271,631 & 0,8957 & 0,0136 & \\
\hline
\end{tabular}

C1 - Spray Microjet/Plasnova; C2 - Conector para microtubo/Amanco; C3 - Click Tif-PC/NaanDan; C4 -Katif/Plastro.

Na Figura 8, apresenta-se a relação entre o coeficiente de carga cinética e o índice de obstrução, para todas as combinações tubo-emissor analisadas, utilizando os dados das Tabelas 4 e 5. A equação ajustada revelou um coeficiente de determinação de 95,56\%, significativo ao nível de $5 \%$ de probabilidade. Apresenta-se também o intervalo de predição com 95\% de confiança, contendo todos os pares de pontos experimentais. A equação gerada resultou em $K=1,228 I O^{0,507}$. 


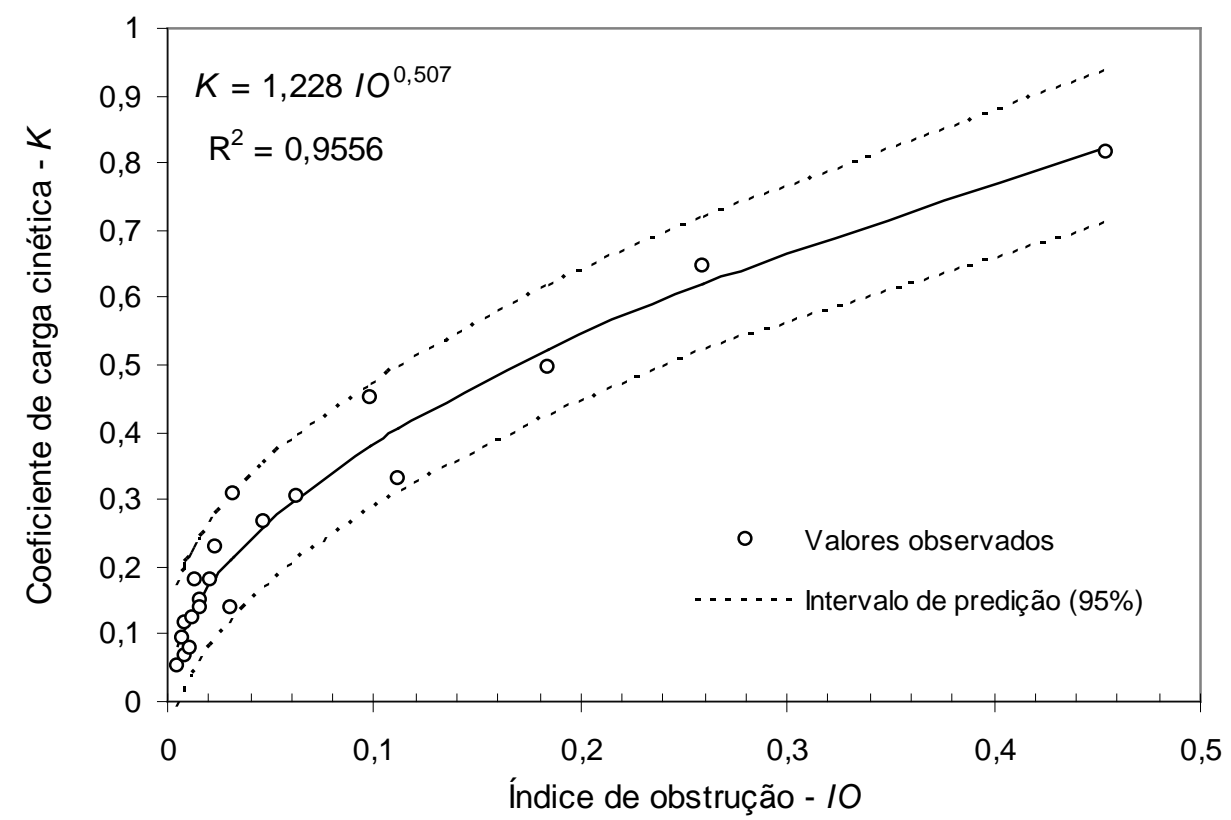

FIGURA 8. Relação entre o coeficiente de carga cinética e o índice de obstrução. Relationship between the coefficient of kinetic head loss and the obstruction index.

\section{CONCLUSÕES}

Para os cinco modelos de tubos avaliados nesta pesquisa, foi encontrada uma equação do tipo potência, semelhante à equação de Blasius, com a constante $b$ igual a 0,300 , para o expoente $m$ fixado em 0,25.

Pela relação do coeficiente de carga cinética $(K)$, utilizado para o cálculo da perda de carga localizada $\left(h f_{L}\right)$, nos quatro modelos de conectores instalados nos cinco diâmetros de tubos, com o índice de obstrução $(I O)$ do conector do emissor, foi gerado um modelo do tipo potência com constantes iguais a 1,228 e expoente no valor de 0,507. O coeficiente de determinação do modelo é de $95,56 \%$.

\section{REFERÊNCIAS}

BAGARELLO,V.; FERRO, V.; PROVENZANO, G.; PUMO, D. Experimental study on flowresistance law for small-diameter plastic tipes. Journal of Irrigation and Drainage Engineering, New York, v.121, n.5, p.313-316, 1995.

BAGARELLO, V., FERRO,V., PROVENZANO, G., PUMO, D. Evaluating pressure losses in dripirrigation lines. Journal of irrigation and Drainage Engineering, New York, v.123, n.1, p.1-7, 1997.

CAMARGO, A. P.; SENTELHAS, P. C. Avaliação do desempenho de diferentes métodos de estimativa da evapotranspiração potencial no estado de São Paulo, Brasil. Revista Brasileira de Agrometeorologia, Santa Maria, v.5, n.1, p.89-97, 1997.

CARDOSO,G. G. G. de. Índice geométrico na determinação da perda de carga localizada em conexão de emissores sobre tubos de polietileno de pequenos diâmetros. 2007. 64f. Dissertação (Mestrado em Agronomia-Irrigação e Drenagem) - Escola Superior de Agricultura Luiz de Queiroz, Universidade de São Paulo, Piracicaba, 2007.

CARDOSO, G. G. G.; FRIZZONE, J. A. Perda de carga em linhas laterais de microirrigação. Revista Brasileira de Engenharia de Biossistemas, Campinas, v.1, n.2, p.147-162, 2007. 
MONTGOMERY, D. C.; RUNGER, G. C. Estatística aplicada e probabilidade para engenharia. 2. ed. Rio de Janeiro: Livros Técnicos e Científicos Editora, 2003. P. 120-198p. 2003.

RETTORE NETO, O.; FRIZZONE, J. A.; MIRANDA, J. H.; BOTREL, T. A. Perda de carga localizada em emissores não coaxiais integrados a tubos de polietileno. Engenharia Agrícola, Jaboticabal, v.29, n.1, p.28-39, 2009.

VILELA, L. A. A.; SOCCOL, O. J.; GERVÁZIO, E. S.; FRIZZONE, J. A.; BOTREL, T. A. Alteração no diâmetro de tubos de polietileno submetidos a diferentes pressões. Revista Brasileira de Engenharia Agrícola e Ambiental, Campina Grande, v.7, n.1, p.182-185, 2003.

VON BERNUTH, R. D. Simple and accurate friction loss equation for plastic pipe. Journal of Irrigation and Drainage Engineering, New York, v.116, n.2, p.294-298, 1989.

WILLMOTT, C. J. On the validation of models. Physical Geography, v.2, n.2, p.184-195, 1981. YITAYEW, M. Simplified method for sizing laterals with two or more diameters. Journal of Irrigation and Drainage Engineering, New York, v.135, n.1, p.111-114, 2009. 